\title{
Non-idiopathic peripheral facial palsy: prognostic factors for outcome
}

\author{
Katharina Geißler ${ }^{1,2}$. Elisabeth Urban ${ }^{1} \cdot$ Gerd F. Volk $^{1,2} \cdot$ Carsten M. Klingner $^{2,3} \cdot$ Otto W. Witte ${ }^{2,3}$. \\ Orlando Guntinas-Lichius ${ }^{1,2}$
}

Received: 12 May 2020 / Accepted: 23 September 2020 / Published online: 6 October 2020

(c) The Author(s) 2020

\begin{abstract}
Objectives There is a lack of data on patients' and diagnostic factors for prognostication of complete recovery in patients with non-idiopathic peripheral facial palsy (FP).

Methods Cohort register-based study of 264 patients with non-idiopathic peripheral FP and uniform diagnostics and standardized treatment in a university hospital from 2007 to 2017 (47\% female, median age: 57 years). Clinical data, facial grading, electrodiagnostics, motor function tests, non-motor function tests, and onset of prednisolone therapy were assessed for their impact on the probability of complete recovery using univariable and multivariable statistics.

Results The most frequent reason for a non-idiopathic peripheral FP was a reactivation of Varicella Zoster Virus (VZV; $36.4 \%)$. Traumatic origin had a higher proportion of complete FP (52.9\%). Furthermore, in traumatic FP, the mean interval between onset and start of prednisolone therapy was longer than in other cases (5.6 \pm 6.2 days). Patients with reactivation of VZV, Lyme disease or otogenic FP had a significant higher recovery rate ( $p=0.002, p<0.0001, p=0.018$, respectively), whereas patients with post-surgery FP and other reasons had a significant lower recovery rate $(p<0.0001)$. After multivariate analyses voluntary activity in first EMG, Lyme disease and post-surgery cause were identified as independent diagnostic and prognostic factors on the probability of complete recovery (all $p<0.05$ ).

Conclusion Infectious causes for non-idiopathic FP like VZV reactivation and Lyme disease had best probability for complete recovery. Post-surgery FP had a worse prognosis.
\end{abstract}

Level of evidence 2

Keywords Facial nerve $\cdot$ Paralysis $\cdot$ Paresis $\cdot$ Prognosis $\cdot$ Electrodiagnostics $\cdot$ Stapedius reflex $\cdot$ Recovery

\section{Introduction}

Peripheral facial palsy (FP) is a most common cranial nerve damage presented to otolaryngologists, neurologists, general practitioners, pediatricians. The most frequent diagnosis for FP remains idiopathic FP (Bell's palsy; 70\%) [1], i.e.

Electronic supplementary material The online version of this article (https://doi.org/10.1007/s00405-020-06398-6) contains supplementary material, which is available to authorized users.

Katharina Geißler

katharina.geissler@med.uni-jena.de

1 Department of Otorhinolaryngology, Jena University Hospital, Am Klinikum 1, 07747 Jena, Germany

2 Facial Nerve Center, Jena University Hospital, Jena, Germany

3 Department of Neurology, Jena University Hospital, Jena, Germany a diagnosis of exclusion. Bell's palsy has an incidence of 10-40 per 100.000 [2]. The complete recovery rate for Bell's palsy after onset of prednisolone treatment within $72 \mathrm{~h}$ in the two milestone phase III trials was about $85-94 \%$ within 9-12 months [3, 4].

In contrast, less is known on the outcome of non-idiopathic FP. Temporal bone fractures and facial wounds are a common cause for traumatic FP (10-23\%, [1]). Furthermore, iatrogenic lesions during otologic, parotid and vestibular schwannoma surgery are reasons for postoperative FP [1].

Ramsay Hunt syndrome (4.5-7\%) due to geniculate ganglionitis by reactivation of Varicella Zoster Virus (VZV) is another common diagnosis of non-idiopathic peripheral FP. Outcome is much worse than in Bell palsy [5].

Acute otitis media, cholesteatoma and necrotizing otitis externa can also cause FP [1]. An important cause is Lyme disease. In endemic areas, Lyme disease causes up to $10-25 \%$ of all cases of FP [6]. To complete, malignant 
tumors and rare genetic syndromes are other reasons for nonidiopathic FP $[1,7]$.

Beginning with 2007, the diagnostic and therapeutic procedures including a standardized therapy regime for all patients with acute FP were conformed in the Departments of Otorhinolaryngology and Neurology of Jena University Hospital, Germany. A prospective data registration of these patients was started. In the present study, we analyzed the recovery rates for the different causes and identified prognostic factors of complete recovery.

\section{Methods}

\section{Ethical considerations}

The institutional ethics committee approved the study protocol for a data collection of routine and anonymized hospital data.

\section{Study design and patients}

A standardized prospective data collection was started in 2007 in the Departments of Otorhinolaryngology and Neurology of Jena University Hospital, Germany. The present study focused on patients with non-idiopathic peripheral FP in the acute phase. All patients received an otorhinolaryngologic examination including ultrasound of head and neck and a neurological examination. Laboratory tests included serum and cerebrospinal fluid (CSF) and were analyzed for $\operatorname{IgM}$ and $\operatorname{IgG}$ antibodies against borrelia, varicella zoster virus (VZV), herpes simplex virus (HSV) using enzymelinked immunosorbent assays (ELISA).

In case of a positive borrelia ELISA, a borrelia IgM and IgG line immunoblot assay was performed. Two or more diagnostic criteria were needed to fulfill the diagnosis of Lyme borreliosis: Recent erythema migrans, borrelia antibodies in serum or CSF, CSF pleocytosis $>5 \mathrm{WBC} / \mathrm{mm}^{3}$, $\mathrm{CSF} / \mathrm{serum}$ index $>1.5$.

The presence of mucocutaneous vesicles in the external auditory canal, on the tympanic membrane or on the base of the tongue, or IgM or IgA antibodies against VZV or HSV detected in serum qualified for classification as virally associated FP.

Eight hundred and six patients (805) patients with peripheral FP were admitted between January 2007 and December 2017 , i.e. within a period of 11 years. One hundred and seventy-three patients (173), presenting for the first time after $>2$ months after onset, were excluded. All patients with Bell's palsy (368) were excluded. A causal origin was already known or could be found in 264 patients. These patients were included in this study.
All patients were hospitalized. Treatment followed the underlying disease and the German guideline for the treatment of FP [11]. As symptomatic treatment, a tapered course of oral corticosteroids over 7 days was regarded as standard treatment [11]. This conservative treatment was given to all patients. In case of VZV reactivation, the patients received additionally acyclovir for 5 days, in case of Lyme disease ceftriaxone for 7 days.

\section{Diagnostics and main outcome measures}

\section{FP grading system}

On the day of first presentation, the FP was graded by the admitting physician according to the House-Brackmann (HB) six-point facial grading system [8], and also according to the Stennert index $[9,10]$. The Stennert index classifies the face at rest $(0-4$ points; $0=$ normal to $4=$ complete loss of resting tone) and during motion ( $0-6$ points; $0=$ normal to $6=$ no motion), separately. Clinically, the palsy was defined as complete if the patient presented with a complete loss of motor function in the affected hemiface or if the palsy deteriorated to a complete palsy during the inpatient course of treatment. Otherwise, the palsy was defined as incomplete palsy.

\section{Electrodiagnostics}

The electrodiagnostic tests to evaluate the facial motor function are described in detail elsewhere [12]. Briefly, baseline electroneurography (ENoG), blink reflex testing (BR) and needle electromyography (EMG) were performed as early as possible. If first EMG was performed earlier than 14 days after onset, the examination was repeated at least one time and later than 14 days after onset. The following categorized diagnostic criteria were evaluated: ENoG, peak-to-peak amplitude loss of $>30 \%$ (yes $=$ pathologic/no); BR, no R1 and R2 responses ipsilateral (yes/no); EMG, pathological spontaneous activity (yes/no), only single fiber activity or no activity during voluntary EMG (yes/no).

\section{Additional functional tests}

The other facial nerve function tests were categorized as follows: Ipsilateral stapedius reflex test normal (yes/no); complete loss of gustatory function on ipsilateral side using three-drop-test (yes/no); lacrimal function by Schirmer test $(<10 \mathrm{~mm} / \geq 10 \mathrm{~mm})$; vestibular function represented by normal caloric stimulation (yes/no), and pure tone audiometry for hearing test (normal/ipsilateral hearing loss).

Follow-up examinations included always an otorhinolaryngologic and an EMG examination. Two physicians examined all patients. The first follow-up examination was 
performed after 6 weeks and then every 3 months until complete recovery. In case of incomplete recovery, recovery with defective healing, the patients were followed in 3 -month intervals until pathological spontaneous activity disappeared and/or the patients did not show any improvement for further 3 months.

\section{Statistical analysis}

If not indicated otherwise, data are presented with mean values \pm standard deviation (SD). All statistical analyses were performed using IBM SPSS, version 25 . Primary outcome criterion was complete recovery of non-idiopathic FP defined as a grade I on the HB scale indicating normal facial function. Kaplan-Meier curves were constructed to calculate the probability of complete recovery over the time (Fig. 1). The prognostic influence of the diagnostic variables on recovery was analyzed with the log-rank test. Prognostic factors associated with significant impact on complete recovery with a probability value of $<0.05$ were included in multivariate analyses using Cox's proportional hazards model. The hazard ratio of the multivariate Cox regression model indicated the probability of complete recovery. The significance level for the multivariable analyses was set at $p<0.05$.

\section{Results}

\section{Patients' and facial palsy characteristics}

264 patients were included. The median age was 57 years. The gender ratio was balanced (47\% female and 53\% male patients). There was no significant side predominance (48.1\% right and $51.9 \%$ left side). Two-thirds were classified at baseline to have an incomplete palsy $(59.5 \%)$ and one-third a complete palsy (31.4\%). The median score on HB scale at baseline was III. The median Stennert indexes at rest and during voluntary activity at baseline were 2 and 4 , respectively. $17 \%$ of the patients had a diabetes mellitus. The most often reason for a non-idiopathic FP was a herpes zoster oticus (36.4\%). FP was occurred after surgery in $25.4 \%$ (mainly vestibular schwannoma surgery: $9.5 \%$; parotid surgery: $8.0 \%$ ) of the cases. Lyme disease $(11.7 \%)$, otogenic reasons $(7.2 \%)$, trauma $(6.4 \%)$ and other diagnoses $(12.9 \%)$ were other reasons for non-idiopathic FP (Table 1). Patients
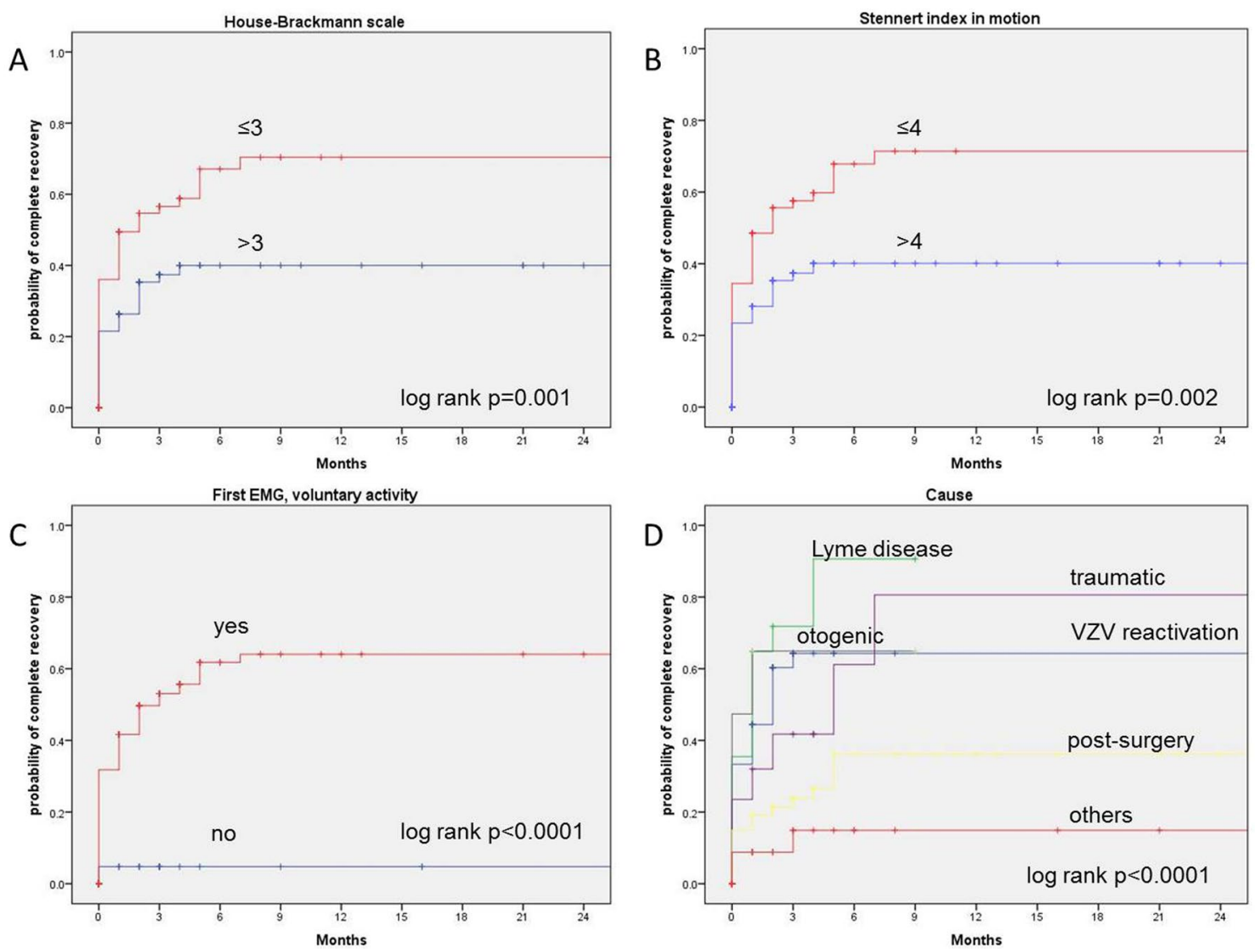

Fig. 1 Kaplan-Meier curves showing parameters with prognostic influence on the probability of complete recovery. a House-Brackmann scale; b stennert index in motion; c first EMG, voluntary activity; $\mathbf{d}$ causes of non-idiopathic FP 
with trauma, often after temporal bone fracture with lateonset-palsy, as underlying disease had the highest rate complete FP $(52.9 \%, p=0.016)$, i.e. the highest rate of severe cases. In connection with this, traumatic FP was associated more often with pathology spontaneous activity in the first EMG examination $(35.3 \%, p<0.0001)$ and less often with voluntary activity in the first EMG examination (58.8\%) in comparison to the other reasons (Table 1).

$70.5 \%$ of the patients received a prednisolone therapy. In traumatic FP, the interval between start of prednisolone therapy and onset of FP was longer $(5.6 \pm 6.2$ days, $p=0.038)$ than in other causes. Patients with otogenic FP were younger $(42.1 \pm 22.2$ years, $p=0.005)$ than the others (Table 1$)$.

On average, House-Brackmann scale and the Stennert index at rest improved after the mean follow-up time of $9.6 \pm 18.0$ months $(p<0.001$, Table S1).

\section{Influence of patients' and diagnostic results on the recovery time}

The median recovery time was 5 months. $39.8 \%$ showed a complete recovery, $32.2 \%$ a partial recovery and $9.8 \%$ no recovery, respectively (18.2\% data missed). Higher initial House-Brackmann grading $(p=0.001)$, higher initial Stennert index in motion $(p=0.002)$, no voluntary activity in first EMG $(p<0.0001)$ and the some causes of FP $(p<0.0001)$ were negative predictors for complete recovery (Table S2).

\section{Influence of patients' diagnosis on the recovery time}

Patients with reactivation of VZV, Lyme neuroborreliosis or otogenic peripheral FP had a significant higher recovery rate $(p=0.002, p<0.0001, p=0.018)$, whereas patients with FP after surgery and other reasons had a significant lower recovery rate $(p<0.0001$, Table S3).

\section{Multivariate analysis}

After multivariate analyses, voluntary activity in first EMG, Lyme disease and post-surgery cause were identified as independent diagnostic and prognostic factors on the probability of complete recovery (Table 2), in which Lyme disease is associated with better and post-surgery cause with worse recovery rate.

\section{Discussion}

\section{Synopsis of key/new findings}

In this presented cohort register-based study on prognostic factors for complete recovery of non-idiopathic FP the most often reason for a non-idiopathic peripheral FP was an infection with Varicella Zoster Virus (36.4\%). In 25.4\% of the cases, FP was occurred after surgery. Lyme disease $(11.7 \%)$, otogenic $(7.2 \%)$, traumatic $(6.4 \%)$ and other diagnosis (12.9\%) were less frequently reasons. Traumatic reasons had a higher proportion of complete facial palsies (52.9\%), associated more often with pathology spontaneous activity in the first EMG examination (35.3\%) and less often with voluntary activity in the first EMG examination (58.8\%). Furthermore, in traumatic FP the interval with onset of prednisolone therapy was longer $(5.6 \pm 6.2$ days). After analysis the recovery time to normal facial function the onset of prednisolone therapy, the House-Brackmann scale at begin, the Stennert index in motion at begin, voluntary activity in first EMG and cause of FP had influence on the recovery time. Patients with infection with VZV, Lyme neuroborreliosis or otogenic peripheral FP had a significant higher recovery rate in comparison to non-idiopathic FP, whereas patients with FP after surgery and other reasons had a significant lower recovery rate. Lyme disease is associated with better and post-surgery cause with worse recovery rate.

\section{Strengths and weaknesses of the study}

A strength of the study is the consequent registration of all new patients with non-idiopathic peripheral FP treated in one tertiary university hospital in a register with standardized data set since 2007. All cases were treated together by the department of otorhinolaryngology and the department of neurology in the Facial Nerve Center of the university hospital. Another strength is the comprehensive diagnostic work-up (not only for prognostic reasons but primarily also for differential diagnostics) including the most relevant electrodiagnostic tests and other tests originally used as topodiagnostic tests in the era before introduction of highresolution radiologic imaging. As facial grading is subjective and has a limited reliability [13], it is important to notice that EMG was performed as a standard diagnostic tool, too. EMG allows for an objective measurement of facial muscle function [12]. The importance was confirmed by the present study. EMG was more reliable to predict outcome than facial grading.

Patients were not treated within a randomized prospective clinical trial. Therefore, a selection bias cannot be ruled out. The duration of follow-up of the patients was variable. No defined times of investigation during the follow-up for all patients, like in a prospective clinical trial, were available. To overcome this limitation, Kaplan-Meier statistics were used to determine the probability of complete recovery over time. 
European Archives of Oto-Rhino-Laryngology (2021) 278:3227-3235

3231
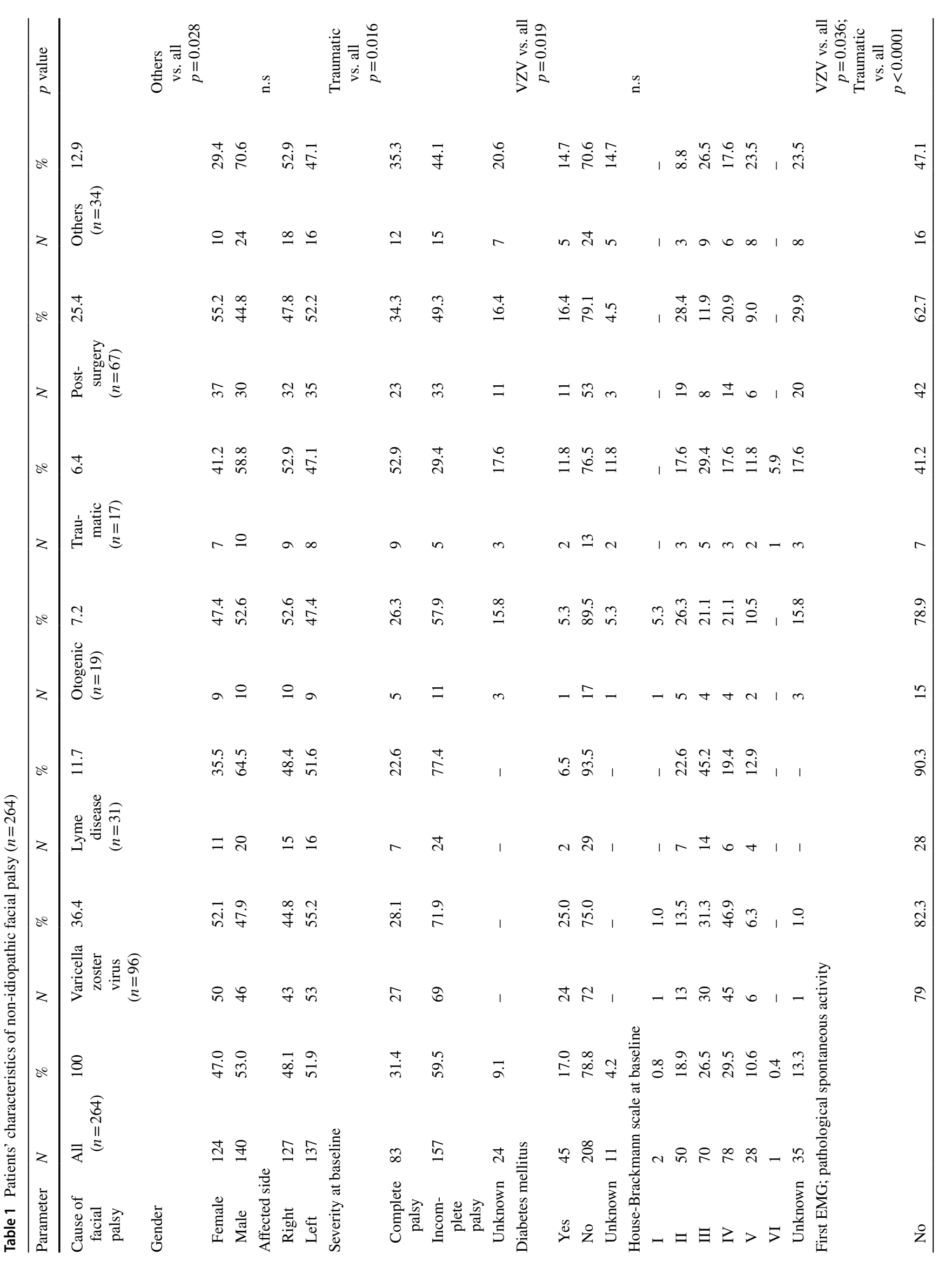

Springer 


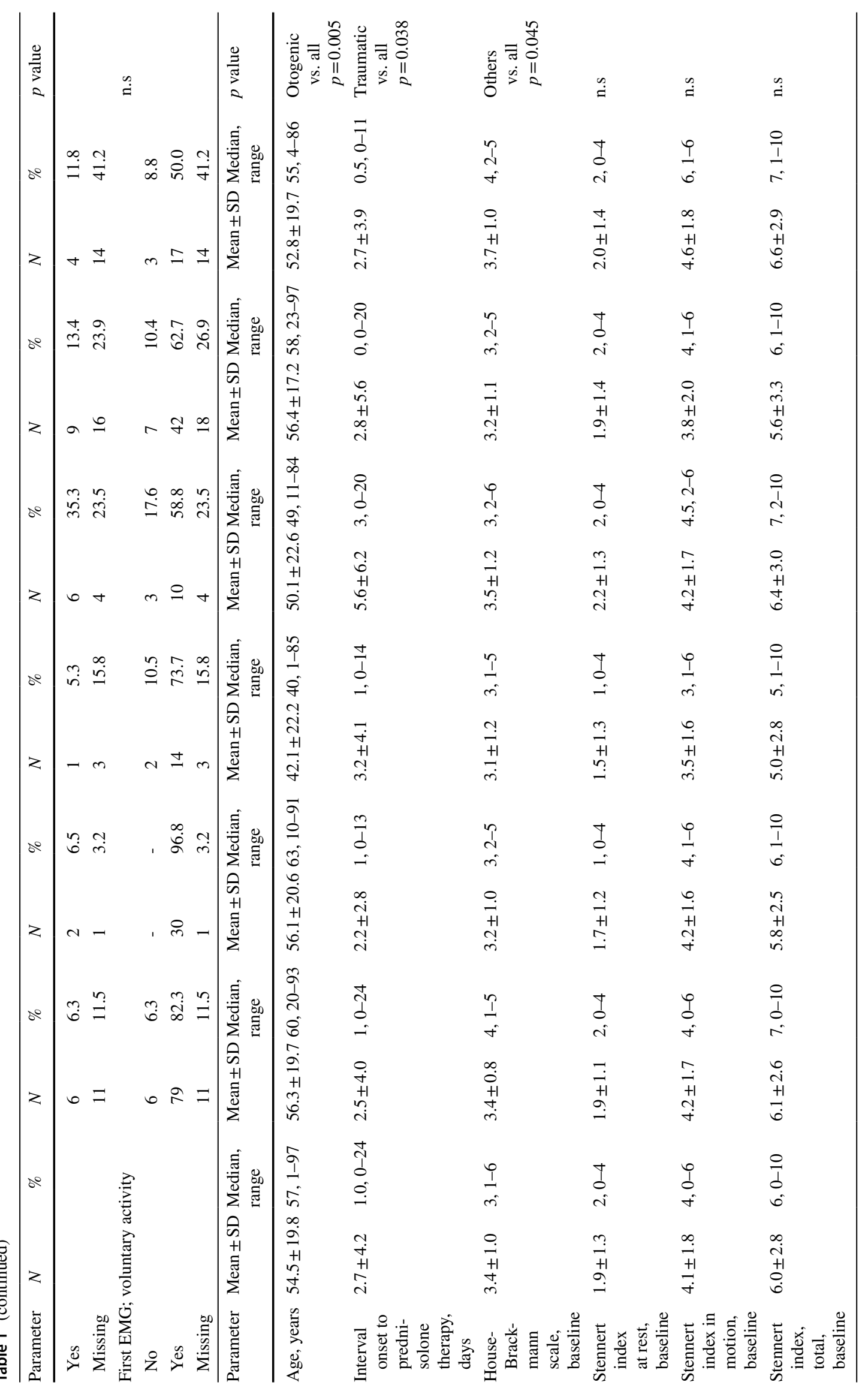


Table 2 Multivariate Cox regression analyses on independent diagnostic and prognostic factors on the probability of complete recovery

\begin{tabular}{|c|c|c|c|c|}
\hline Parameter & Hazard ratio & $95 \%$ CI lower & $95 \%$ CI upper & $p$ value* \\
\hline \multicolumn{5}{|l|}{ Model 1} \\
\hline First EMG; voluntary activity & & & & 0.039 \\
\hline No & 1 & & & \\
\hline Yes & 8.156 & 1.115 & 59.651 & \\
\hline House-Brackmann scale (median) & & & & 0.916 \\
\hline$>3$ & 1 & & & \\
\hline$\leq 3$ & 1.042 & 0.489 & 2.218 & \\
\hline Stennert index, in motion (median) & & & & 0.280 \\
\hline$>4$ & 1 & & & \\
\hline$\leq 4$ & 1.499 & 0.719 & 3.124 & \\
\hline Varicella zoster reactivation & & & & 0.183 \\
\hline No & 1 & & & \\
\hline Yes & 1.349 & 0.868 & 2.096 & \\
\hline \multicolumn{5}{|l|}{ Model 2} \\
\hline First EMG; voluntary activity & & & & $\mathbf{0 . 0 3 7}$ \\
\hline No & 1 & & & \\
\hline Yes & 8.284 & 1.134 & 60.522 & \\
\hline House-Brackmann scale (median) & & & & 0.685 \\
\hline$>3$ & 1 & & & \\
\hline$\leq 3$ & 0.856 & 0.405 & 1.811 & \\
\hline Stennert index, in motion (median) & & & & 0.153 \\
\hline$>4$ & 1 & & & \\
\hline$\leq 4$ & 1.711 & 0.820 & 3.570 & \\
\hline Lyme disease & & & & $\mathbf{0 . 0 3 5}$ \\
\hline No & 1 & & & \\
\hline Yes & 1.752 & 1.040 & 2.951 & \\
\hline \multicolumn{5}{|l|}{ Model 3} \\
\hline First EMG; voluntary activity & & & & 0.044 \\
\hline No & 1 & & & \\
\hline Yes & 7.708 & 1.057 & 56.187 & \\
\hline House-Brackmann scale (median) & & & & 1.000 \\
\hline$>3$ & 1 & & & \\
\hline$\leq 3$ & 1.000 & 0.464 & 2.156 & \\
\hline Stennert index, in motion (median) & & & & 0.261 \\
\hline$>4$ & 1 & & & \\
\hline$\leq 4$ & 1.543 & 0.724 & 3.288 & \\
\hline Post-surgery palsy & & & & 0.022 \\
\hline No & 1 & & & \\
\hline Yes & 0.460 & 0.236 & 0.896 & \\
\hline
\end{tabular}

CI confidence interval

*Significant $p$ values in bold

\section{Comparisons with other studies}

It is important to compare the outcome of non-idiopathic FP to the outcome of idiopathic FP: EMG evaluation for pathological spontaneous activity is in non-idiopathic FP and also in Bell's palsy [14] an important prognostic factor for recovery time from acute facial palsy. Volk et al. identified moreover start of treatment, grading and stapedius reflex test. In this study, otogenic cause (87\%), Lyme disease (78\%), VZV reactivation (77\%) had a slightly better probability of recovery at 9 months after onset in comparison to Bell`s palsy (73\%). Traumatic cause had a worse outcome (46\%) [14]. Furthermore, Urban et al. identified severity of the palsy, facial electrodiagnostics and stapedius reflex testing are the most powerful tool for prognostication of recovery time after Bell's palsy. Prednisolone therapy, 
which started within a time window of $96 \mathrm{~h}$ after onset, had the highest probability of complete recovery [15].

Chang et al. analyzed the characteristics of patients with acute peripheral FP showing Varicella Zoster Virus DNA in saliva [16] and showed, that VZV DNA-positive patients had worse hearing and incomplete recovery compared to VZV DNA-negative patients. In our collective, patients with VZV infection had significant higher recovery rate in comparison to all other non-idiopathic facial palsies. Morishima et al. evaluated 2013 prognostic factors of synkinesis following Bell's palsy and Ramsay Hunt syndrome [17]. The lowest Yanagihara score, the change in Yanagihara score after 1 month and ENoG value at the onset were found to be important factors for predicting synkinesis. Takemoto et al. identified $2011 \mathrm{ENoG}$ as the most effective factor for prediction of the prognosis of peripheral FP in case of Bell's palsy or Ramsay Hunt syndrome, and more than $85 \%$ degeneration had the best specificity and sensitivity to predict nonrecovery [18]. In our study, voluntary activity in first EMG was the most valid independent factor for recovery. Kim et al. and Cai et al. realized that patients with Ramsay Hunt syndrome had a significant unfavorable recovery rate and poorer prognosis than patients with Bell's palsy [19, 20]. In our collective, patients with Ramsay Hunt syndrome had the same recovery rate as patients with Bell's palsy.

Jowett et al. demonstrated, that there was an association between corticosteroid use in acute Lyme disease associated FP and worse long-term facial function outcomes [21]. In our opinion, nevertheless the cause of non-idiopathic FP a fast treatment with prednisolone after onset could be useful and essential.

Ping et al. analyzed possible prognosis factors of systemic steroid in managing traumatic facial nerve palsy after a blunt craniofacial injury retrospectively [22]. The outcome showed steroid therapy onset within $24 \mathrm{~h}$ and steroid therapeutic duration for longer than 14 days possessed a significantly better recovery rate. In our study, steroid started late and could potentially had influence on the recovery rate. Yadav et al. evaluated the outcome of conservative management in traumatic facial paralysis with regard to type of trauma, onset and electrodiagnostic tests [23]. Patients with incomplete FP are candidates for conservative management and also patients with complete facial paralysis for up to 3 months even in cases where ENoG and NET suggest poor prognosis. In our study, patients with traumatic peripheral FP had no significant worse recovery rate than patients with other causes of non-idiopathic FP. Thakar et al. concluded 2018, that for undisplaced temporal bone fractures, nonsurgical treatment leads to near complete recovery and is superior to reported surgical results [24]. Surgical exploration should not be first-line treatment for undisplaced longitudinal temporal bone fractures associated with complete facial nerve paralysis and unfavorable electrophysiological features. In our opinion, surgery remains reserved for special cases. In most cases, conservative therapy with fast onset of therapy with prednisolone is sufficient. An immediate total facial paralysis associated with a clear-cut fracture going through the Fallopian canal (Canalis nervi facialis) is perhaps the only case requiring surgery after traumatic FP in cause of temporale bone fracture [25]. In case of direct cut through branches of facial nerve, a fast reconstruction is necessary and the therapy of first choice [7, 14].

Especially early-onset FP after vestibular schwannoma surgery had a bad prognosis of recovery [26]. In case of post-surgery FP, spontaneous activity and voluntary activity in first EMG could be an essential tool to differentiate patients' prognosis. In our opinion and experience, if there is no recovery within 1 year after surgery a facial nerve reconstruction should be considered.

\section{Clinical applicability of the study}

Traumatic reasons of non-idiopathic peripheral FP seem to be associated with severe disease histories. The higher proportion of diagnosis of degenerative axonal lesion could be one point of worse outcome. Furthermore, the late onset of prednisolone therapy could be another. Especially in case of traumatic reasons, a fast onset of prednisolone therapy and/ or facial nerve decompression by surgery should be considered. It would be worthwhile to analyze if patients with worse prognosis would profit from an intensified therapy (higher prednisolone dose). The addition of the EMG result is very helpful to identify as early as possible the patients with high risk for incomplete recovery and development of synkinesis. Because of the functional and psychosocial impairments that might occur, these patients need early education about treatment options, and short follow-up visit intervals. Still many patients with facial synkinesis are undertreated. High-risk patients might profit from an early referral to a specialist center for facial nerve care [27].

\section{Conclusion}

In our study infectious causes for non-idiopathic FP like VZV reactivation and Lyme disease had best probability for complete recovery. Post-surgery FP had a worse prognosis.

Author contributions OGL developed the idea for the study. KG and OGL made all drafts of the manuscript. EU and GFV administered the database. KG and OGL performed the multivariable statistics. All authors analyzed and interpreted the data. All authors revised the manuscript. KG wrote the first draft of the manuscript. No honorarium, grant, or other form of payment was given to anyone to produce the manuscript. Each author listed on the manuscript has seen and 
approved the submission of this version of the manuscript and takes full responsibility for the manuscript.

Funding Open Access funding enabled and organized by Projekt DEAL. The authors received no specific funding for this work.

Data availability All authors had full access to all of the data in the study. OGL takes responsibility for the integrity of the data and the accuracy of the data analysis. No additional data available.

\section{Compliance with ethical standards}

Conflict of interest The authors have declared that no competing interests exist. The authors have no financial interest to declare in relation to the content of this article.

Open Access This article is licensed under a Creative Commons Attribution 4.0 International License, which permits use, sharing, adaptation, distribution and reproduction in any medium or format, as long as you give appropriate credit to the original author(s) and the source, provide a link to the Creative Commons licence, and indicate if changes were made. The images or other third party material in this article are included in the article's Creative Commons licence, unless indicated otherwise in a credit line to the material. If material is not included in the article's Creative Commons licence and your intended use is not permitted by statutory regulation or exceeds the permitted use, you will need to obtain permission directly from the copyright holder. To view a copy of this licence, visit http://creativecommons.org/licenses/by/4.0/.

\section{References}

1. Plumbaum K, Volk GF, Boeger D, Buentzel J, Esser D, Steinbrecher A, Hoffmann K, Jecker P, Mueller A, Radtke G, Witte OW, Guntinas-Lichius O (2017) Inpatient treatment of patients with acute idiopathic peripheral facial palsy: a population-based healthcare research study. Clin Otolaryngol 42:1267-1274

2. De Diego-Sastre JI, Prim-Espada MP, Fernández-García F (2005) The epidemiology of Bell's palsy. Rev Neurol 41(5):287-290

3. Swan IR, Donnan PT, Morrison JM, Smith BH, McKinstry B, Davenport RJ, Vale LD, Clarkson JE, Hammersley V, Hayavi S, McAteer A, Stewart K, Daly F (2007) Early treatment with prednisolone or acyclovir in Bell's palsy. N Engl J Med 357(16): 1598-1607

4. Engström M, Berg T, Stjernquist-Desatnik A, Axelsson S, Pitkäranta A, Hultcrantz M, Kanerva M, Hanner P, Jonsson L (2008) Prednisolone and valaciclovir in Bell's palsy: a randomised, double-blind, placebocontrolled, multicentre trial. Lancet Neurol 7(11):993-1000

5. Lee HY, Kim MG, Park DC, Park MS, Byun JY, Yeo SG (2012) Zoster sine herpete causing facial palsy. Am J Otolaryngol 33(5):565-571

6. Halperin JJ, Golightly M (1992) Long island neuroborreliosis collaborative study group lyme borreliosis in Bell's palsy. Neurology 42:1268-1270

7. Finkensieper M, Volk GF, Guntinas-Lichius O (2012) Facial nerve disorders. Laryngorhinootologie 91(2):121-141 (quiz 142)

8. House JW, Brackmann DE (1985) Facial nerve grading system. Otolaryngol Head Neck Surg 93(2):146-147

9. Stennert E, Limberg CH, Frentrup KP (1977) An index for paresis and defective healing-an easily applied method for objectively determining therapeutic results in facial paresis (author's transl). HNO 25(7):238-245
10. Guntinas-Lichius O, Straesser A, Streppel M (2007) Quality of life after facial nerve repair. Laryngoscope 117(3):421-426

11. Heckmann JG, Lang C, Glocker FX, Urban P, Bischoff C, Weder B, Reiter G, Meier U, Guntinas-Lichius O, Association of the Scientific Medical Societies in Germany (AWMF) (2012) The new S2k AWMF guideline for the treatment of Bell's palsy in commented short form. Laryngorhinootol 91(11):686-692

12. Grosheva M, Wittekindt C, Guntinas-Lichius O (2008) Prognostic value of electroneurography and electromyography in facial palsy. Laryngoscope 118(3):394-397

13. Fattah A, Gurusinghe A, Gavilan J, Hadlock TA, Marcus JR, Marres H, Nduka CC, Slattery WH, Snyder-Warwick AK, Sir Charles Bell Society (2015) Facial nerve grading instruments: systematic review of the literature and suggestion for uniformity. Plast Reconstr Surg 135:569-579

14. Volk GF, Klingner C, Finkensieper M, Witte OW, GuntinasLichius O (2013) Prognostication of recovery time after acute peripheral facial palsy: a prospective cohort study. BMJ Open 3(6): 003007

15. Urban E, Volk GF, Geißler K, Thielker J, Dittberner A, Klingner C, Witte OW, Guntinas-Lichius O (2020) Prognostic factors for the outcome of Bells' palsy: a cohort register-based study. Clin Otolaryngol. https://doi.org/10.1111/coa.13571

16. Chang DS, Lee J, Shin SA, Lee HY (2018) Characteristics of patients with acute peripheral facial palsy showing varicella zoster virus DNA in saliva. J Med Virol Actions 90(5):959-964

17. Morishima N, Yagi R, Shimizu K, Ota S (2013) Prognostic factors of synkinesis after bell's palsy and ramsay hunt syndrome. Auris Nasus Larynx 40:431-434

18. Takemoto N, Horii A, Sakata Y, Inohara H (2011) Prognostic factors of peripheral facial palsy: multivariate analysis followed by receiver operating characteristic and Kaplan-Meier analyses. Otology \& Neurotology 32:1031-1036

19. Kim SH, Jung J, Jung SY, Dong SH, Byun JY, Park MS, Kim SH, Yeo SG (2019) Comparative prognosis in patients with ramsayhunt syndrome and bell's palsy. Eur Archiv Oto-Rhino-Laryngol 276:1011-1016

20. Cai Z, Li H, Wang X, Niu X, Ni P, Zhang W, Shao B (2017) Prognostic factors of Bell's palsy and Ramsay Hunt syndrome. Medicine 96:2

21. Jowett N, Gaudin RA, Banks CA, Hadlock TA (2017) Steroid use in lyme disease-associated facial palsy is associated with worse long-term outcomes. Laryngoscope 127:1451-1458

22. Lee PH, Chi-Cheng Liang CC, Shiang-Fu Huang SF, Han-Tsung Liao HT (2018) The outcome analysis of traumatic facial nerve palsy treated with systemic steroid therapy. J Craniofac Surg 29(7):1842-1847

23. Yadav S, Panda NK, Verma R, Bakshi J, Modi M (2018) Surgery for post-traumatic facial paralysis: are we overdoing it? Eur Arch Otorhinolaryngol 275:2695-2703

24. Thakar A, Gupta MP, Srivastava A, Agrawal D, Kumar A (2018) Nonsurgical treatment for posttraumatic complete facial nerve paralysis. JAMA Otolaryngol Head Neck Surg 144(4):315-321

25. Darrouzet V, de Bonfils-Dindart C, Bébéar JP (1998) Management of post-traumatic facial paralysis. A decision based on a series of 85 cases. Neurochirurgie 44(4):235-246

26. Chang S, Makarenko S, Despot I, Dong C, Westerberg BD, Akagami R (2020) Differential recovery in early- and late-onset delayed facial palsy following vestibular schwannoma resection. Oper Neurosurg (Hagerstown) 18(1):34-40

27. Butler DP, Morales DR, Johnson K, Nduka C (2019) Facial palsy: when and why to refer for specialist care. B J Gen Pract 69(688):579-580

Publisher's Note Springer Nature remains neutral with regard to jurisdictional claims in published maps and institutional affiliations. 